\title{
Laser cooling techniques: standard and alternated optical molasses
}

\author{
Naomy D. Gomes*1, Mônica A. Caracanhas ${ }^{1}$, Kilvia M. Farias ${ }^{1}$, Vanderlei S. Bagnato ${ }^{1}$ \\ ${ }^{1}$ Instituto de Física de São Carlos, Universidade de São Paulo, São Carlos, São Paulo, SP, Brasil
}

Received on March 30, 2017. Accepted on April 24, 2017.

\begin{abstract}
The technique of laser cooling, whose basic idea was proposed in 1975 [1, provided the development of many important areas like high-resolution spectroscopy [2, atomic clocks $[3]$ and quantum gases confined in magnetic or optical traps [4,5]. Among the techniques applied to cooling neutral atoms is the optical molasses 6 ] that will be explored in this work. Here we study the standard molasses and a new alternated molasses configuration, which consists of turning on just one pair of counter-propagating laser beams per cycle, and alternating this pair between the three spatial directions. We analyse the two techniques under the lens of the radiation force, by exploring a semiclassical treatment, in order to obtain the minimum energy attained by each of them at the end of the cooling process. We compare, then, these two cooling techniques, pointing out their advantages.
\end{abstract}

Keywords: Laser cooling, Optical molasses, Semiclassical treatment, Doppler limit

\section{Introduction}

The development of the laser cooling and trapping methods for neutral atoms were essential to the progress of many fields. The reduction of the random thermal velocities using radioactive forces allowed the study of the atomic collisions and the determination of the atom inner structure with great accuracy 7]. Associated to the magneto-optical trap 8] (MOT), this technique also formed the basis for the achievement of Bose-Einstein condensation in atomic gases [9], opening the way to a deeper understanding of the quantum-physical behaviour of gases at ultra-low temperatures.

In this Letter we describe the standard and the alternated optical molasses techniques, first introducing the concept of radiation force, making use of a semiclassical treatment to consider the interaction of electromagnetic radiation with the atomic system. This force is at the heart of the cooling technique, since it has the effect of dissipating the energy of the atoms and, thus, cooling them.

We determine the evolution of the atomic energy during the cooling process, for the standard and alternated molasses configurations.

By looking at the evolution of energy and its minimum, we can trace parallels between the two techniques and their advantages/disadvantages concerning the experimental process and the atomic densities reached by each one of them.

A gas of bosonic atoms enters in the quantum-degenerate regime and forms a Bose-Einstein condensate (BEC) if

*Endereço de correspondência: naomy.gomes@usp.br its spatial density $\rho$ exceeds a critical value, which is given approximately by the relation $\rho \lambda_{d B}^{3}=2.612$, where $\lambda_{d B}=h / \sqrt{2 \pi m k_{B} T}$ is the thermal de Broglie wavelength, $T$ is the temperature, $\mathrm{h}$ and $k_{B}$ are the Planck's and Boltzmann's constant, respectively, and $m$ the mass of an atom [10]. Low temperatures in combination with high densities have to be reached to obtain quantum degeneracy.

Since the early days of laser cooling, it has been asked if the quantum degenerate regime could be reached using this efficient method as the only cooling process. Until now, however, laser cooling has to be followed by evaporative cooling procedure 5] to produce quantum degeneracy in cold atomic samples. The re-absorption of photons scattered during laser cooling [1] induces an effective repulsion and heating of the low energetic atoms. That is a severe obstacle to the required density be reached.

As will be shown, the alternated molasses configuration may be a better cooling technique in what concerns the spatial density of the atomic sample.

\section{Radiation force}

There are basically two kinds of forces exerted on an atomic system due to interaction with radiation: spontaneous and dipole forces $[7,11$. The spontaneous force originates from the momentum transferred to the atom during the photon absorption or emission processes. Each photon transfers to the atom a momentum $\hbar k$ in a time interval related to the laser intensity, laser detuning and atomic excited state lifetime. Otherwise, the dipole force comes from the interaction of atomic electric dipole, that 
is, the induced electronic transition, with the gradient of the radiation intensity.

In this paper we used a semiclassical approach to consider the interaction of the radiation with the atomic system 12,13, that is, we considered atoms, which will be treated quantum mechanically, interacting with a classical electromagnetic field. This theory, which is based on Ehrenfest's theorem and optical Bloch equations, have the advantage of giving an unified treatment to the radiation force, including effects of spontaneous emission and the induced dipole interaction, maintaining the simplicity of the most elementary theories. The atom considered in this theory has two energy levels (two-level quantum system).

Considering the atom moving sufficiently slow $(\mathbf{r}(t))$, i.e., electric field amplitude $\left(E_{0}\right)$ and phase time derivative $(\dot{\theta}(t)=\nabla \theta(\mathbf{r}(t)) \cdot \dot{\mathbf{r}}(t))$ varying a small fraction during atomic excitation lifetime $\left(\Gamma^{-1}\right)$, the force due to the interaction with the radiation will be given by

$$
\mathbf{F}=-\frac{\hbar \Gamma \Omega^{2} \nabla \theta+\hbar(\Delta+\dot{\theta}) \nabla \Omega^{2}}{4(\Delta+\dot{\theta})^{2}+\Gamma^{2}+2 \Omega^{2}},
$$

where $\Omega=\mu E(\mathbf{r}(t)) / \hbar$ is the Rabi frequency, which characterizes the coupling between the atom and the radiation; $\Delta=\omega-\omega_{0}$ is the detuning between laser frequency $\omega$ and atomic resonant frequency $\omega_{0}=\left(E_{1}-\right.$ $\left.E_{2}\right) / \hbar$, the latter proportional to the energy distance between the two energy levels; $\Gamma$ is the spontaneous emission rate and $\theta$ corresponds to the phase of electromagnetic field. Considering a plane wave with wavevector $\mathbf{k}$ and frequency $\omega$, the electromagnetic field is given by $\mathbf{E}(\mathbf{r}(t))=\mathbf{E}_{0} \cos (\mathbf{k} \cdot \mathbf{r}-\omega t)$. We have, then, a constant Rabi-frequency $\Omega=\mu E_{0} / \hbar$ and the field phase at the atom position is $\theta(\mathbf{r})=-\mathbf{k} \cdot \mathbf{r}$, with $\dot{\theta}(\mathbf{r})=-\mathbf{k} \cdot \dot{\mathbf{r}}$. For this particular condition, the radiation force reduces to the spontaneous force

$$
\begin{aligned}
\mathbf{F} & =\frac{\Gamma \Omega^{2} \hbar \mathbf{k}}{4(\Delta-\mathbf{k} \cdot \dot{\mathbf{r}})^{2}+\Gamma^{2}+2 \Omega^{2}} \\
& =\frac{\Gamma}{2} \hbar \mathbf{k} \frac{s_{0}}{\left(2 \frac{\Delta-\mathbf{k} \cdot \dot{\mathbf{r}}}{\Gamma}\right)^{2}+1+s_{0}}
\end{aligned}
$$

where $s_{0}=\frac{2 \Omega^{2}}{\Gamma^{2}}=\frac{I}{I_{0}}$ is the saturation parameter at resonance $(\Delta=0)$, which is related to the laser intensity $I$ and limits the force magnitude. According to equation (2), the spontaneous force saturates at the maximum value $F_{\text {sat }}=\frac{\Gamma}{2} \hbar k$, since the atom can not cycle between excited to ground state faster than $\Gamma / 2$. The first term in the denominator of equation (2) is velocity dependent and, as the atom slows down, the Doppler shift $\omega_{D}=$ $-\mathbf{k} \cdot \dot{\mathbf{r}}$ brings it out of the resonance condition, lowering the force magnitude. In order to reach the deceleration that changes the atomic speed by hundreds of $\mathrm{m} / \mathrm{s}$, it is necessary to compensate the Doppler shift to keep $\Delta+\omega_{D}<<\Gamma$, either modifying $\omega_{D}$ or $\Delta[11$.

Consider now two laser beams in the same direction ( $z$ direction - figure (1) ), but in opposite sense (which we will call senses + and - ). Within a low laser intensity regime, the resulting force in the atomic system will be just the sum of the spontaneous force of each beam $F_{z}=F_{+}+F_{-}$. For small atomic velocities, we can expand the sum to first order in $v_{z}$

$$
F_{z}=8 \hbar k^{2} \frac{|\Delta|}{\Gamma} \frac{s_{0}}{\left(1+4 \Delta^{2} / \Gamma^{2}+s_{0}\right)^{2}} v_{z},
$$

where $v_{z}$ is the atomic velocity in the laser direction and $F_{x}=F_{y}=0$. For $\Delta<0$, that is, $\omega<\omega_{0}$, there will be a dissipative viscous force acting in the $z$ direction, reducing the atom velocity.

Writing this damping force as $F_{z}=-\alpha v_{z}$, we have the damping coefficient

$$
\alpha=8 \hbar k^{2} \frac{|\Delta|}{\Gamma} \frac{s_{0}}{\left(1+4 \Delta^{2} / \Gamma^{2}+s_{0}\right)^{2}} .
$$

This force has a maximum value $F_{z}=-\frac{\hbar k^{2}}{2} v_{z}$ that occurs for the saturation parameter $s_{0}=2$ and detuning $\Delta=-\frac{\Gamma}{2}$. It is important to notice that equation 3 is valid only for low laser intensity regime [6], since we consider the saturation term to be simply $\bar{I} / I_{0}$, which would be equivalent to assume that each wave saturates the atom independently. For higher intensities, the laser beams can not be considered independently anymore. Effects like stimulated emission have to be considered [11], besides the dipole force due to the standing waves [14].

\section{Optical molasses}

As showed in the previous section, the combination of the opposite laser beams produces a viscous force that dissipates the energy of the atom to the electromagnetic field. To determine the total energy of the atomic system, however, we should consider the quantized nature of electromagnetic field to evaluate the contribution of the spontaneous emission process 15], where an atom initially in an excited state can return to the ground state even in the absence of incident radiation (see figure (2)).

In spite of this random process does not change the mean atomic momentum $(\langle\Delta P\rangle=0)$, since the phase and the direction of propagation of the radiation emitted are random, it increases, however, the atomic kinetic energy $\left(\left\langle(\Delta P)^{2}\right\rangle \neq 0\right)$. This additional energy is given

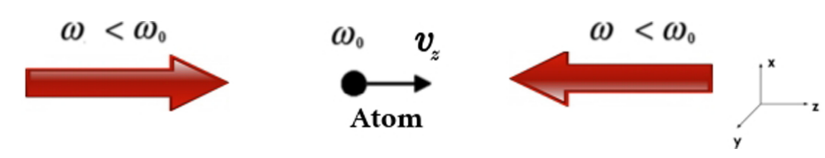

Figure 1: Cooling process: two laser beams in opposite sense with laser frequency $\omega$ smaller than the atomic resonant frequency $\omega_{0}$. 


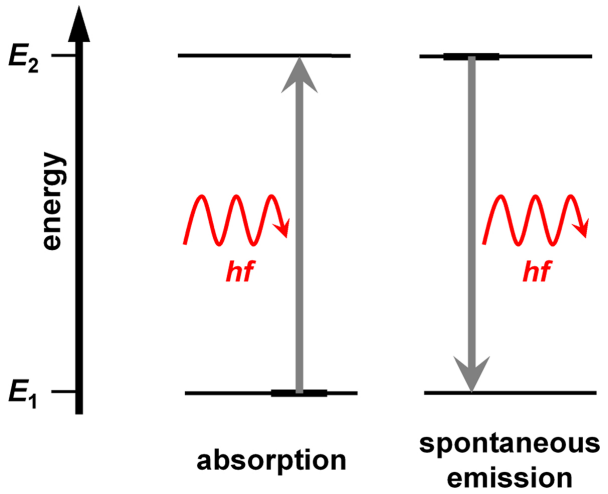

Figure 2: Two level system absorption and spontaneous emission, the latter being a random process.

by the recoil rate

$$
R=2 \frac{\Gamma}{2} \frac{s_{0}}{\left(1+4 \Delta^{2} / \Gamma^{2}+s_{0}\right)} \frac{\left\langle(\Delta P)^{2}\right\rangle}{2 m},
$$

which corresponds to the scattering rate of the laser light by the atom times its average kinetic energy. The latter can be calculated considering the atomic diffusion in the momentum space, with discrete walks of size $\hbar k$ at each absorption or emission process 16], resulting in the following expression

$$
R=\Gamma \frac{s_{0}}{\left(1+4 \Delta^{2} / \Gamma^{2}+s_{0}\right)} \frac{(\hbar k)^{2}}{2 m} \eta .
$$

In equation (6), $\eta=\eta_{a b s}+\eta_{\text {emis }}$ is the stochastic parameter that will be determined for different molasses configurations (1D, 2D and 3D molasses).

We consider an isotropic emission process where $\eta_{\text {emis }}=$ $1 / 3$, that is, the emission will have an equal probability for each direction, independently of the photon absorption direction. For the $1 D$ molasses, the probability of absorption will be $\eta_{a b s}=1$ in the direction of the laser and zero at the other two directions, giving

$$
\begin{aligned}
& R_{i}^{1 D}=\Gamma \frac{s_{0}}{\left(1+4 \Delta^{2} / \Gamma^{2}+s_{0}\right)} \frac{4}{3} \frac{(\hbar k)^{2}}{2 m}, \\
& R_{j}^{1 D}=\Gamma \frac{s_{0}}{\left(1+4 \Delta^{2} / \Gamma^{2}+s_{0}\right)} \frac{1}{3} \frac{(\hbar k)^{2}}{2 m},
\end{aligned}
$$

where $i$ represents the direction of the pair of laser beams and $j$ the other directions without the laser. Otherwise, for $2 D$ molasses, the probability of absorption will be $\eta_{a b s}=1 / 2$ for each laser beam direction. Hence, for this case

$$
\begin{aligned}
R_{i}^{2 D} & =\Gamma \frac{s_{0}}{\left(1+4 \Delta^{2} / \Gamma^{2}+s_{0}\right)} \frac{5}{6} \frac{(\hbar k)^{2}}{2 m}, \\
R_{j}^{2 D} & =\Gamma \frac{s_{0}}{\left(1+4 \Delta^{2} / \Gamma^{2}+s_{0}\right)} \frac{1}{3} \frac{(\hbar k)^{2}}{2 m} .
\end{aligned}
$$

With the $3 D$ molasses, the diffusion will be in three dimensional momentum space, with the absorption probability $\eta_{a b s}=1 / 3$ for each direction, then

$$
R_{i}^{3 D}=\Gamma \frac{s_{0}}{\left(1+4 \Delta^{2} / \Gamma^{2}+s_{0}\right)} \frac{2}{3} \frac{(\hbar k)^{2}}{2 m} .
$$

The balance between the energy lost due to dissipative cooling force and that acquired in the recoil process allow us to construct the dynamic equation for the atomic kinetic energy

$$
\begin{array}{r}
\frac{d K_{i}^{N}}{d t}=v_{i} F_{i}+R_{i}^{N}=\alpha \frac{2 K_{i}}{m}+R_{i}^{N}, \\
\frac{d K_{j}^{N}}{d t}=R_{j}^{N} .
\end{array}
$$

In Eqs. (10), the indices $\mathrm{N}$ classifies the 1D , 2D and 3D molasses configuration, for the direction with $(i)$ and without $(j)$ laser illumination, which solutions will be explored in the following.

Solving these equations for $N=1$, i.e., for $1 D$ molasses, with $\alpha$ giving by equation (4) and $R^{1 D}$ by equation (7), we obtain

$$
\begin{aligned}
K_{i}^{1 D}(t) & =e^{-t / \tau}\left(K_{0}-\xi\right)+\xi, \\
K_{j}^{1 D}(t) & =K_{0}+\frac{1}{4} \beta t,
\end{aligned}
$$

where $K_{0}$ is the initial kinetic energy in the directions $i$ and $j$. The parameter $\tau=\frac{m}{2 \alpha}$ represents the characteristic time for the cooling process, $\beta=\Gamma \frac{s_{0}}{\left(1+4 \Delta^{2} / \Gamma^{2}+s_{0}\right)} \frac{4}{3} \frac{(\hbar k)^{2}}{2 m}$ is the rate that the kinetic energy increases due to the spontaneous emission and $\xi=\tau \beta$ is the heating in the characteristic time interval $\tau$. The kinetic energy of the atom in the $1 D$ molasses configuration will be the sum of these solutions

$$
K^{1 D}(t)=2 K_{0}+\xi+e^{-t / \tau}\left(K_{0}-\xi\right)+\frac{1}{2} \beta t,
$$

and it is straightforward to verify that it has a minimum value at the time $t_{\text {min }}^{1 D}=\tau \ln \left[2\left(K_{0} / \xi-1\right)\right]$, with

$$
K_{\text {min }}^{1 D}\left(t_{\text {min }}^{1 D}\right)=2 K_{0}+\frac{3}{2} \xi+\frac{1}{2} \beta \tau \ln \left[2\left(K_{0} / \xi-1\right)\right] .
$$

Although the energy reaches a minimum value in the direction $i$ of the laser configuration, on the other hand, as it can be seen from equation (11b), to the other two directions, $j$, the energy increases linearly in time. Then, for time intervals bigger than $t_{m i n}$, the system starts to accumulate energy, since it is transferred to the directions without laser illumination, where we do not have the cooling process to control the recoil. This heating that 
occurs after $t_{\text {min }}$ in the $1 D$ molasses does not occur in $3 D$ laser beam arrangement. For $N=3$, with the force given by equation (3) and the recoil term by equation (9), the differential equation for the total energy can be written as

$$
\frac{d K^{3 D}}{d t}=-\frac{1}{\tau} K^{3 D}+3\left(3 H_{i}^{3 D}\right)=-\frac{1}{\tau} K^{3 D}+\frac{9}{2} \beta .
$$

Equating the energy rate to zero we obtain a stationary condition, where the energy is given by

$$
K_{s}=\frac{9}{2} \xi=\frac{3}{16} \frac{\hbar \Gamma^{2}\left(1+4 \Delta^{2} / \Gamma^{2}+s_{0}\right)}{|\Delta|}
$$

Solving equation (14), in terms of the $K_{s}$

$$
K^{3 D}(t)=e^{-t / \tau}\left(K_{0}-K_{s}\right)+K_{s} .
$$

According to equation (15), the minimum energy value is reached with a laser detuning of $\Delta=-\Gamma / 2$. Then, considering $s_{0}<<1$ in this same equation, we obtain the Doppler cooling limit 7

$$
K_{\min }^{3 D}=\frac{3}{4} \hbar \Gamma
$$

\section{Optical molasses with temporal alternated beams}

In this section we introduce our new cooling technique, to be compared with the standard one, the alternated molasses configuration. It has one pair of lasers at each direction, which, however, will be alternately turned on during a selected time interval. A natural choice is to apply the characteristic time $\tau$ related to the cooling process. In the following we calculate the atomic kinetic energy evolution during the cooling cycles. Using the solution for $1 D$ molasses (equation (11)) we derive the following sequence, starting with the laser pair in $x$

$$
\begin{aligned}
K_{x}(\tau) & =\frac{1}{e}\left(K_{0 x}-\xi\right)+\xi \\
K_{y}(\tau) & =\frac{1}{e}\left(K_{0 y}-\xi\right)+\xi \\
K_{z}(\tau) & =\frac{1}{4} \xi+K_{0 z} .
\end{aligned}
$$

Considering now the lasers at $y$, again turned on during a time interval $\tau$

$$
\begin{aligned}
K_{x}(2 \tau) & =\frac{1}{4} \xi+K_{x}(\tau) \\
& =\frac{1}{e}\left(K_{0 x}-\xi\right)+\frac{5}{4} \xi, \\
K_{y}(2 \tau) & =\frac{1}{e}\left(K_{y}(\tau)-\xi\right)+\xi \\
& =\frac{1}{e}\left(K_{0 y}-\frac{3}{4} \xi\right)+\xi, \\
K_{z}(2 \tau) & =\frac{1}{4} \xi+K_{z}(\tau) \\
& =\frac{1}{2} \xi+K_{0 z} .
\end{aligned}
$$

With the pair along the $z$ direction

$$
\begin{aligned}
K_{x}(3 \tau) & =\frac{3}{2} \xi+\frac{1}{e}\left(K_{0 x}-\xi\right) \\
K_{y}(3 \tau) & =\frac{5}{4} \xi+\frac{1}{e}\left(K_{0 y}-\frac{3}{4} \xi\right) \\
K_{z}(3 \tau) & =\xi+\frac{1}{e}\left(K_{0 z}-\frac{1}{2} \xi\right) .
\end{aligned}
$$

Finally, after finished one cycle, we obtain

$$
K(3 \tau)=\frac{15}{4} \xi+\frac{1}{e}\left(K_{0}-\frac{9}{4} \xi\right) .
$$

By using recurrence relations for the initial kinetic energy, after the second cycle we will have

$$
K(6 \tau)=\frac{15}{4} \xi-\frac{9}{4} \xi \frac{1}{e}+\frac{K(3 \tau)}{e}=\frac{15}{4} \xi+\frac{3}{2} \xi \frac{1}{e}-\frac{9}{4} \xi \frac{1}{e^{2}}+\frac{K_{0}}{e^{2}} .
$$

With the same procedure

$$
K(9 \tau)=\frac{15}{4} \xi+\frac{3}{2} \xi \frac{1}{e}+\frac{3}{2} \xi \frac{1}{e^{2}}+\frac{K_{0}}{e^{2}}-\frac{9}{4} \xi \frac{1}{e^{3}}+\frac{K_{0}}{e^{3}}
$$

This initial sequence allows us to generalize the energy after $n$ cycles

$$
K(3 n \tau)=\frac{15}{4} \xi+\frac{3}{2} \xi \sum_{i=1}^{n-1} \frac{1}{e^{i}}-\frac{9}{4} \xi \frac{1}{e^{n}}+\frac{K_{0}}{e^{n}} .
$$

After many cycles, i.e., for large values of $n$, the second term of equation $(24)$ converges to $\sum_{i=1}^{\infty} \frac{1}{e^{i}} \approx 0,581876$ (the last two terms can be neglected). Then, the energy reaches a minimum value close to the expected limit given by equation 15

$$
K(3 n \tau)=4,62296 \xi \approx \frac{9}{2} \xi
$$




\section{Numerical Simulation}

In this section we compare the atomic kinetic energy evolution resulted from the previous studied molasses configurations. For the simulation, we chose the atomic strontium species, since it has many advantages in the laser cooling context [17]. We selected the isotope ${ }^{84} \mathrm{Sr}$, which has an interest transition linewidth in the $\mathrm{KHz}$ region $(\Gamma / 2 \pi=7.4 \mathrm{KHz})$, allowing the achievement of temperatures in the nanokelvin regime.

Figure 3 shows the atomic energy evolution in the standard $3 D$ molasses and the alternated optical molasses for the atomic vapour initial at $T_{0}=0.001 \mathrm{~K}$. The laser parameters were fixed to the values: $\Delta=-\Gamma / 2$ (minimum energy condition), $\lambda=689 \mathrm{~nm}$ and $s_{0}=2$ (maximum atomic deceleration).

As expected by our calculations, in both cases we have the same energy limit which correspondents to a temperature of $355 \mathrm{nK}$.

\section{Standard Optical Molasses versus Alternated Optical Molasses}

It was shown above that the two techniques reach the same energy limit. While the standard molasses may be experimentally easier to perform when compared to the alternated molasses, since it does not require the alternation of the laser beams, our new configuration can possibly reach a higher phase space density.

The atomic density reached in magneto-optical traps has an upper bond value due to the repulsive forces between the atoms caused by the reabsorption of scattered photons, as stated before.

For a collection of atoms in a optical trap with laser

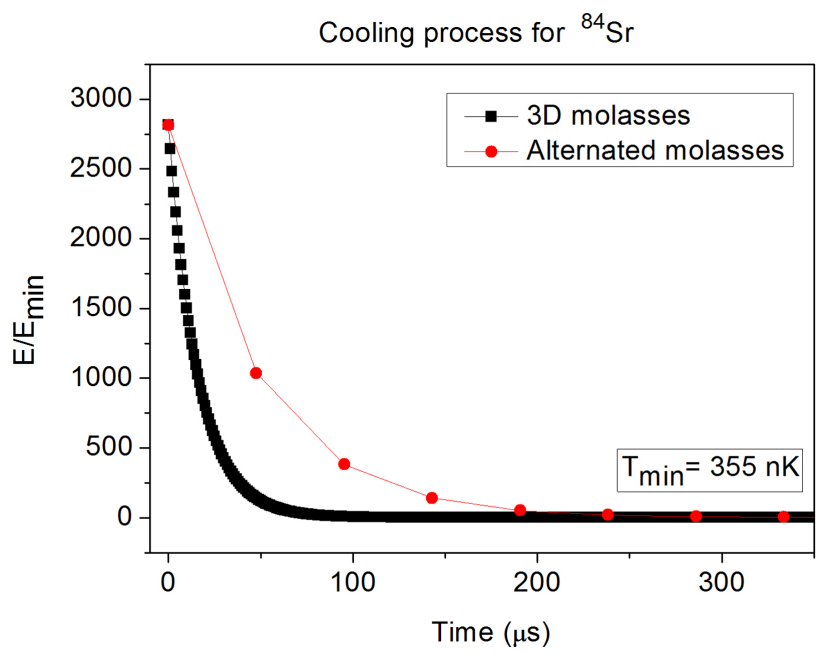

Figure 3: Comparison between the standard $3 D$ and the alternated molasses, showing the energy as a function of time for strontium atoms (energy rescaled by a factor $E_{\min }=\frac{9}{2} \xi$ ), leading to a temperature of $355 \mathrm{nK}$. field intensity $I$, this force obeys the relation 18

$$
\nabla \cdot \mathbf{F}_{R}=6 \sigma_{R} \sigma_{L} I \rho / c
$$

where $c$ is the speed of light, $\sigma_{R}$ and $\sigma_{L}$ are the direct and scattered laser light, respectively, and $\rho$ is the atomic density.

Due to the intensity gradients, the confining lasers also produce an attenuation force $F_{A}$ in the trapped atoms [19, which compress the atomic cloud:

$$
\nabla \cdot \mathbf{F}_{A}=-6 \sigma_{L}^{2} I \rho / c .
$$

These two forces $F_{A}$ and $F_{R}$ must balance the trapping force $-\mathbf{k} . \mathbf{r}$ due to the electromagnetic field in the limit that the temperature can be neglect, which implies a maximum achievable density

$$
\rho_{\max }=\frac{c k}{2 \sigma_{L}\left(\sigma_{R}-\sigma_{L}\right) I} .
$$

Thus, the force generated by the re-absorption of light gives an upper limit to the atomic density that is inversely proportional to the field intensity. Since in the alternated molasses this intensity is three times smaller than in the standard 3D molasses, the maximum density will be higher.

\section{Conclusion}

In this work we studied and compared two configurations of optical molasses. Starting with the semiclassical treatment of the laser-cooling concept, that is, explaining how neutral atoms interact with the radiation force, we determined and studied the dynamic equation that governs the atomic energy in a molasses configuration. This treatment allows us to shown that the atomic sample reaches the same minimum temperature expected for the standard and alternated molasses, without relevant increase in the cooling time. Remarkably, the alternated configuration has the advantage of reducing the power beam fluctuation during the cooling process. This factor is a problematic issue in the standard molasses configuration, that uses three retro-reflected laser beams. Otherwise, the alternated molasses can be produced with just one beam deflected between the three spatial directions, using acoustic-optic modulators with appropriate time interval $(\approx 70 \mathrm{kHz})$ 20. That reduces considerable this source of fluctuation between laser beams.

The main difference of both configurations, however, can be related to the atomic density reached in the magneto-optical traps, which is constraint due to the repulsive forces between the atoms caused by re-absorption of the scattered photons. As a practical matter, the power of the re-scattered light sets a limit to the number of atoms which can be confined in a magneto-optical trap, since the magnitude of this repulsive force depends on the laser field intensity $I$. The alternated molasses will diminish the total light intensity incident on the atom sample 
if compared with the usual 3D molasses, favouring higher atomic density values in the trap. That is an important improvement of this particular method in the direction of bring the atomic sample to the quantum degenerate regime using the laser cooling technique alone.

\section{References}

[1] W.D. Phillips, Rev. Mod. Phys. 70, 721 (1998).

[2] W.M. Itano, J.C. Bergquist, R.G. Hulet and D.J. Wineland, Phys. Rev. A 36, 2220 (1987).

[3] N.F. Ramsey, Metrologia 42, S1 (2005).

[4] S. Chu, Scientific American 266, 71 (1992).

[5] E.A. Cornell and C.E. Wieman, Rev. Mod. Phys. 74, 875 (2002).

[6] P.D. Lett, W.D. Phillips, S.L. Rolston, C.E. Tanner, R.N. Watts and C.I. Westbrook, J. Opt. Soc. Am. B 6, 2084 (1989).

[7] H.J. Metcalf and P. van der Straten, Laser Cooling and Trapping (Springer, New York, 1999).

[8] E. Tilman, I. Bloch and T.W. Hasch, Phys. Rev. A 58, R2664 (1998)

[9] A.J. Leggett, Rev. Mod. Phys. 73, 307 (2001).

[10] S. Stellmer, B. Pasquiou, R. Grimm and F. Schreck, Phys. Rev. Lett. 110, 263003-1-263003-5 (2013).

[11] H.J. Metcalf and P. van der Straten, J. Opt. Soc. Am. B 20, 887 (2003).

[12] R.J. Cook, Phys. Rev. A 20, 224 (1979).

[13] J. Dalibard and C. Tannoudji, J. Opt. Soc. Am. B 2, 1707 (1985).

[14] J. Dalibard, Le Role des Fluctuations dans la Dynamique d'un Atome Couplé au Champ Électromagnétique. Thèse de Doctorat, Université de Paris, Paris, 1986.

[15] R. Loudon, The Quantum Theory of Light (Oxford University Press Inc., New York, 2001), 3rd ed.

[16] V.S. Bagnato and S.C. Zilio, Revista Brasileira de Física 19, 127 (1989).

[17] S. Stellmer, M.K. Tey, B. Huang, R. Grimm and F. Schreck, Phys. Rev. Lett 103, 200401 (2009).

[18] T. Walker, D. Sesko and C. Wieman, Phys. Rev. Lett. 64, 408 (1990).

[19] J. Dalibard, Optics Commun. 68, 203 (1988).

[20] D.J. McCarron, A Guide to Acousto-Optic Modulators (Durham University, Durham, 2007). 